\title{
DESARROLLO DE NUEVAS TECNOLOGÍAS PARA LA COCCIÓN DE ALIMENTOS
}

Responsable: Lic. Hugo Torres Muro Miembro: Bach. Boris Manuel Milla Taco

\section{RESUMEN}

La mitad de la población del mundo utiliza biomasa para cocinar alimentos, así como para calefacción y calentamiento de agua. En general, se usan cocinas a fuego abierto, dispositivos en los que la combustión se da de manera incompleta, provocando emisión de partículas y gases contaminantes, que pueden provocar problemas de salud en los usuarios. En el Perú, alrededor del $25 \%$ de la población utiliza leña como fuente de energía para uso doméstico, principalmente en zonas rurales y altoandinas. (Díaz-Jiménez, 2000).

El presente artículo describe la metodologia para diseñar, construir, evaluar y determinar los beneficios del uso de una cocina mejorada en escuelas rurales de la región, aplicada por el Centro de Energías Renovables de Tacna (CERT) en la comunidad rural de Santa Rita en el distrito de Calana, Tacna.

Los resultados obtenidos en la investigación nos permiten concluir que las estufas mejoradas de leña son una buena opción para mitigar la contaminación del aire en interiores, asi como una alternativa para mejorar las condiciones de preparación de desayunos y almuerzos escolares en las zonas rurales y ahorrar significativamente leña, disminuyendo la creciente deforestación y sus consecuencias globales.

\section{ABSTRACT}

Half of the population of the world, it uses biomass to cook foods, as well as it stops heating and heating of water. In general kitchens are used to open fire, devices in those that the combustion is given in an incomplete way, causing emission of particles and polluting gases that can cause problems of health in the users. In the Peru, around the population's $25 \%$ it uses firewood like energy source for domestic use, mainly in Andean rural and high areas. (DiazJiménez, 2000).

The present article presents the methodology to design, to build, to evaluate and to determine the benefits of the use of a kitchen improved in rural schools of the region, carried out by the Center of Renewable Energy of Tacna (CERT) in Santa Rita's rural community in the district of Calana, Tacna.

The results obtained in the investigation, allow us to conclude that the improved stoves of firewood are a good option to mitigate the contamination of the air in interiors, as well as an alternative to improve the conditions of preparation of breakfasts and school lunches in the rural areas and to save firewood significantly, diminishing the growing deforestation and their global consequences.

\section{INTRODUCCIÓN}

Según el Balance Nacional de Energía en el Perú, realizado por la Oficina Técnica del MEM entre los años 1999 y 2000, se estima que la producción anual de leña fue de 7625000 metros cúbicos, de los cuales la leña aporta $4603 \times 10^{6} \mathrm{~kg}$ de biomasa para el consumo final directo. En Tacna el consumo de leña para 1999 fue de 13260 $\mathrm{m}^{3}$, lo que representa el $0,17 \%$ del consumo total en el Perú que bordeo los $7624690 \mathrm{~m}^{3}$ La leña continúa, predominando en la estructura de consumo, siendo utilizada principalmente en cocción con una eficiencia entre el $5 \%$ y el $15 \%$. La importancia de la leña dentro de los productos energéticos, es tal que su aporte total aproximado es del $30 \%$ de la energia producida a nivel nacional y bastante por encima de las otras fuentes: diesel $19 \%$, electricidad $11 \%$, kerosene $9 \%$.

\subsection{Contaminación en el Interior}

La combustión de la leña en fogones abiertos se da de manera incompleta e incontrolada y genera, por ello, una gran cantidad de particulas y gases contaminantes. En el interior de las viviendas, particularmente en donde se cocina, ocurre la mayor exposición a estos contaminantes, siendo las mujeres y los niños los más expuestos por pasar la mayor parte del tiempo en dicha habitación. Ellos se exponen durante periodos de tres a siete horas diarios, a niveles muy altos de contaminantes, durante muchos años (Albalak, 1997).

De acuerdo con Cooper (1982), la quema de biomasa en fogones abiertos genera diecisiete sustancias consideradas "contaminantes prioritarios" por la Agencia de Protección 
Ambiental de los Estados Unidos (EPA), para las cuales existen evidencias de toxicidad; más de catorce compuestos carcinógenos; seis tóxicos para los cilios y agentes muco-coagulantes; y cuatro precursores del cáncer. En los fogones tradicionales de los paises en desarrollo las concentraciones de partículas superan ampliamente las concentraciones consideradas como permisibles de acuerdo con normas internacionales establecidas para la calidad del aire en ambientes exteriores.

La exposición a contaminantes emitidos por fogones abiertos puede producir efectos adversos sobre la salud; sus efectos dependen de factores como (Smith, 1987): emisión, concentración, exposición, dosis, vulnerabilidad. Existe evidencia consistente de que la contaminación intramuros debida a la utilización de biomasa (leña) al interior de la vivienda aumenta significativamente el riesgo de infecciones respiratorias agudas en niños y de enfermedades pulmonares crónicas en adultos (WHO, 2002).

\subsection{Cocinas o Estufas Mejoradas}

Se han realizado muchos intentos para mejorar la combustión en las estufas que utilizan biomasa, tanto para que haya una menor emisión de humo como para que se utilice menos combustible. En la región Tacna, especificamente en las provincias de Candarave, Tarata y Tacna, el Centro de Energías Renovables de Tacna (CERT) ha trabajado desde el año 2000 en la difusión de estufas eficientes de leña, las mismas que han sido adaptadas a las condiciones y necesidades de la población local.

El principio de funcionamiento de una cocina mejorada se basa en la combustión completa de la leña, transfiriendo toda esta energía calorífica directamente a la olla donde se encuentran los alimentos, pero con la diferencia de que todo el humo que se genera internamente es expulsado por una chimenea hacia el exterior del lugar donde se está cocinando. En una cocina mejorada se busca reducir las pérdidas de calor optimizando el recinto incandescente (aislándolo) y colocando apropiadamente las ollas, a la vez se acondiciona una chimenea, la que dirige el humo residual hacia el exterior y promueve un tiro de la llama, que se controla con compuertas adecuadas.

\subsection{Partes de una Cocina Mejorada}

- Caja de Fuego. Es el recinto donde está contenida la leña y ocurre la combustión. Debe ser suficientemente grande para acomodar el tamaño y tipo de leños usados, pero suficientemente angosta para dirigir el fuego debajo de la olla. La olla debe estar tan cerca de la fuente de calor para absorberlo, pero no tan cerca que ahogue el fuego.

- Entrada a la Caja de Fuego. Es por donde se alimenta de combustible a la estufa y por donde penetra el aire. El tamaño y disposición de la entrada afectan el arreglo estructural del fuego. La entrada debe ser suficientemente ancha para permitir fácil acceso del fuego, y permitir la colocación cruzada de los leños.

- Compuerta Reguladora. Es la puerta que controla el flujo de aire y da acceso a la leña. Una puerta frontal, colocada antes del fuego, controla el flujo de aire a la caja de fuego. Una puerta trasera, colocada en el túnel al final de los agujeros de las ollas o en la chimenea, controla el tiro a través de la estufa. Las compuertas pueden fabricarse de láminas de metal, losetas de barro o de concreto. La puerta debe cerrar tan herméticamente como sea posible. La compuerta trasera controla aún más el flujo del aire al ajustar el tiro de la chimenea. Después que se extingue el fuego se cierran las compuertas, para conservar el calor y los alimentos calientes, por largo tiempo

- Túmulos. Los túmulos son obstrucciones al flujo de aire y gases calientes. Aumentan la turbulencia y dirigen el flujo del aire hacia las ollas. El espacio entre el fondo de la olla y el túmulo debe ser lo mínimo requerido para mantener un tiro apropiado.

- Chimenea. La chimenea saca el humo de la cocina, esta fuerza con la que jala el humo a lo largo de la chimenea se llama tiro. Ocurre porque los gases calientes del fuego son más livianos que el aire circundante, y por tanto suben llevando consigo una corriente de aire caliente a lo largo de la estufa. La fuerza del tiro también succiona aire por la entrada a la caja de fuego, y cualquier rajadura de la estufa.

- Hornillas. Desde nuestra perspectiva, la hornilla se considera como una máquina térmica. La eficiencia de la hornilla refleja la relación entre la cantidad de energía empleada para cocer los alimentos y la energía total generada al quemar la leña.

\section{MATERIALESYMÉTODOS}

\subsection{Diseño de la Cocina Mejorada}

Esta cocina se diseñó teniendo en cuenta el modelo Lorena, pero haciendo algunos cambios de acuerdo con las necesidades y costumbres características de las personas que iban a usarla. 


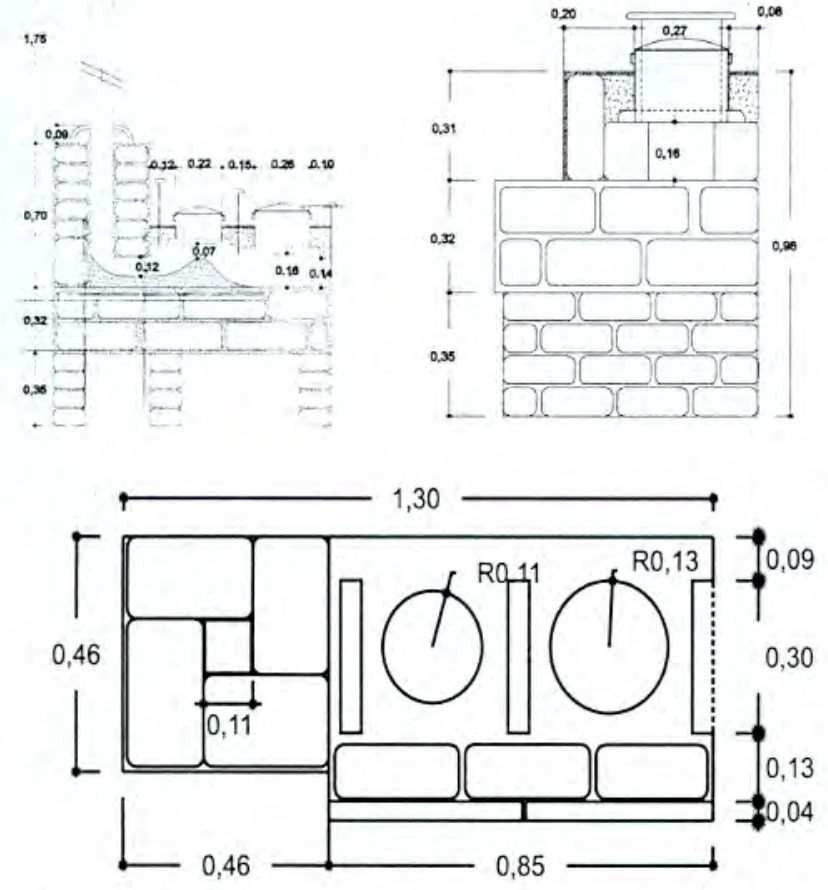

FIGURA 1 Vista transversal, de frente y de planta de la cocina diseñada; se observan cada una de sus partes y las medidas respectivas (en metros), para su construcción.

\subsection{Construcción de la Cocina Mejorada:}

Para construir la cocina se debe tener en cuenta un procedimiento que va desde seleccionar los materiales adecuados y la forma como se tiene que ir construyendo cada una de sus partes, entre las que podemos citar las siguientes:

- Selección del barro. No se necesita arcilla pura, cualquier suelo barroso puede funcionar si contiene poco sedimento y puede quemarse bien. Hay que cavar profundo para encontrar los barros más puros.

- Selección de la arena. Casi cualquier arena puede funcionar bien, aunque se prefieren arenas con partículas entre 0,5 y $4 \mathrm{~mm}$ de diámetro. La arena con mucho sedimento puede causar problemas.

- Mezclado. El objetivo es distribuir el barro uniformemente sobre la arena. El barro seco debe ser pulverizado y los terrones deben retirarse por medio de tamiz de $4 \mathrm{~mm} 0$ menos.

- Agregado del agua. Si la mezcla está seca aun, se agrega el agua por pocos hasta que el total de la mezcla esté húmedo. La mezcla debe ser homogénea, evitando dejar cualquier trozo de barro.

- Preparación de la base. Se construye una base sólida para la estufa, ya sea sobre el nivel del suelo o arriba de éste. Se usan bloques de concreto, ladrillos, adobes, rocas o cualquier material disponible localmente para levantar la base.

- Construcción del bloque. Se prepara la mezcla como una pasta compacta, lo suficientemente blanda para esparcirse, pero lo suficientemente seca para poder hacer una pila de $10 \mathrm{~cm}$ sin que se deslice. Se coloca la mezcla sobre la base por medio de una pala, una cuchara de albañil o un pedazo de cartón o madera, en capas de 3 a $8 \mathrm{~cm}$ de espesor. En esta etapa, no alisar la superficie o hacerla regular; las capas ásperas ligan mejor.

- Ranuras para la compuerta. La cocina o estufa tiene dos compuertas en los diseños sin chimenea, una al frente y otra atrás. Pueden cortarse de lámina metálica, deslizándose verticalmente a lo largo de corredores adosados al frente de la estufa.

- Excavación de túneles. Se excava manejando cucharas metálicas, grandes machetes o cuchillos grandes. Se mantienen todas las herramientas húmedas, para minimizar la fricción y el desmoronamiento del bloque.

- Encajando las ollas. A menos que se deje deliberadamente un espacio alrededor de las ollas, éstas deben encajar ajustadamente y las bases donde descansan las ollas deben ser firmes y lisas.

- Túmulos. Se construyen debajo de cada hornilla para dirigir los gases calientes hacia el fondo de la olla. Se agrega una pequeña cantidad de la mezcla de Lorena directamente debajo de la hornilla sobre el túnel, a manera de elevarlo hasta $5 \mathrm{~cm}$ del fondo de la olla. En la caja de fuego no debe haber túmulo.

- Terminando la estufa. Las paredes internas de la caja de fuego y los túneles deben bruñirse con el revés de una cuchara $u$ otro objeto redondo.

- Revestimiento de la superficie. Para su protección contra la lluvia o líquidos derramados, puede revestirse la estufa con diversas sustancias impermeables o estéticas.

- La chimenea. Donde los terremotos no son problema, puede construirse de bloques de arena/barro fundidos en un molde pequeño $y$ secados completamente, usando una mezcla con más barro para mortero.

\subsection{Evaluación Técnica}

El balance energético permite encontrar la distribución de energía calorifica incidente sobre 
la olla, su transformación en energía útil y las pérdidas térmicas. Para un intervalo de tiempo, según el principio de conservación de la energía aplicado al sistema del la cocina,se establece que:

$$
Q_{u}=Q_{a b}\left(Q_{p 1}+Q_{p 2}\right)
$$

Donde:

$Q_{u} \quad$ : Energía útil extraída por el agua

$Q_{a b^{*}}$ : Calor absorbido por la olla

$Q_{p 1}$ : Energía perdida por aumento de energía almacenada en la misma cocina

$\mathbf{Q}_{\mathrm{p} 2}$ : Energia perdida por la chimenea

La energía absorbida, útil y perdida en forma de calor tiene las mismas unidades, y se da en Watts hora (Wh). Asimismo la energía útil en una cocina será la energía calorífica "Qu" que gana el agua:

$$
\begin{aligned}
E_{u} & =Q_{u} \\
Q_{u} & =m_{\mathrm{e}} \cdot C_{\mathrm{e}}\left(T_{f}-T_{j}\right)
\end{aligned}
$$

Donde:

$Q_{u} \quad$ :Energía calorífica del agua en (kcal).

$m_{a} \quad$ :Masa del agua en $(\mathrm{kg})$

$C_{\text {e }}$ : Calor especifico del agua $\left(\mathrm{kcal} / \mathrm{kg} .{ }^{\circ} \mathrm{C}\right)$

Asimismo la energía generada por la combustión de la leña se conoce como la energía incidente:

Donde:

$$
E_{i}=m_{1} \cdot P_{c}
$$

$E_{i}$ : Es la energía incidente ( $\left.\mathrm{kWh}\right)$

$\boldsymbol{m}_{\boldsymbol{l}}$ : Masa de leña en $(\mathrm{kg})$

$\boldsymbol{P}_{\mathrm{c}}$ : Poder calorífico de la leña $(\mathrm{kcal} / \mathrm{kg})$. El poder calorífico de la leña es de $3702 \mathrm{Wh} / \mathrm{kg}$

La eficiencia de una cocina a leña esta representada por:

Donde:

$$
\eta=\frac{E_{u}}{E_{i}} \times 100 \%
$$

$\mathrm{E}_{\mathrm{u}}$ : Es la energia útil que extrae el agua $(\mathrm{kJ} / \mathrm{kg})$.

$E_{i}$ :Es la energía incidente causada por la combustión de la leña $(\mathrm{kJ} / \mathrm{kg})$.

El diseño experimental consiste en evaluar comparativamente las cocinas tradicional $y$ mejorada. La evaluación consistió en tomar datos de temperatura del agua desde antes de iniciar la combustión hasta finalizar la combustión del ultimo trozo de leña que queda, midiendo a la vez las temperaturas del ambiente, de perdidas en los costados de las ollas y en la chimenea para evaluar el comportamiento, tanto de la curva de calentamiento, enfriamiento, como el de la eficiencia de ambas cocinas y conocer el tiempo que demora cada uno en llevar el agua a su máxima temperatura.
Para proceder a evaluar ambas cocinas (tradicional y mejorada) comparativamente, es necesario hacerlo bajo las mismas condiciones de operación.

\section{RESULTADOS}

\begin{tabular}{|c|c|c|c|c|c|c|c|c|c|c|c|c|c|}
\hline \multicolumn{8}{|c|}{$\begin{array}{c}\text { COCINA } \\
\text { TRADICIONAL }\end{array}$} & \multicolumn{6}{|c|}{ COCINA MEJORADA } \\
\hline \multirow[t]{2}{*}{$\mathrm{N}^{0}$} & \multirow{2}{*}{$\begin{array}{l}\text { Diempo } \\
\begin{array}{l}\text { Inicio } \\
\text { (h) }\end{array}\end{array}$} & \multirow{2}{*}{\begin{tabular}{c|} 
Tamb \\
$\left({ }^{\circ} \mathrm{C}\right)$ \\
\end{tabular}} & \multicolumn{5}{|c|}{ OLLAN 1} & \multicolumn{3}{|c|}{ OLLAN 1} & \multicolumn{3}{|c|}{ OLLAN 2} \\
\hline & & & $\begin{array}{c}\mathrm{m} \\
(\mathrm{kg})\end{array}$ & $\mathrm{m}_{(\mathrm{kg})}$ & $\left.{ }^{\circ}{ }^{\top}\right)^{\top}$ & ${ }^{\top}{ }^{\top} C^{\prime}$ & $(\mathrm{kg})$ & $\mathrm{m}_{(\mathrm{kg})}^{\mathrm{m}}$ & $\begin{array}{r}T^{T_{i}} \\
\left.\left({ }^{C}\right)^{\prime}\right)\end{array}$ & $\begin{array}{c}T_{f} \\
\left({ }^{\circ} C\right)\end{array}$ & $\underset{(\mathrm{kg})}{\mathrm{m}}$ & $\begin{array}{r}T_{i} \\
\left({ }^{C} \mathrm{C}\right)\end{array}$ & $\left.{ }^{\circ} \mathrm{C}\right)$ \\
\hline 1 & 09:05 & 18 & 0.9 & 8 & 15 & 73 & 0.4 & 4 & 15 & 45 & 4 & 15 & 88 \\
\hline 2 & 11:06 & 21,5 & 1,25 & 8 & 17 & 96 & 0.6 & 3 & 17 & 90 & 5 & 17 & 95 \\
\hline 3 & 13:00 & 23 & 1,5 & 10 & 19,5 & 92 & 0,75 & 4 & 19,5 & 88 & 6 & 19,5 & 95 \\
\hline
\end{tabular}

Los resultados obtenidos para la evaluación térmica comparativa se muestran en la tablas 1 y 2 .

TABLA N ${ }^{\circ}$ 01: Datos obtenidos en la caracterización

\begin{tabular}{|c|c|c|c|c|c|c|c|c|c|c|}
\hline & \multicolumn{4}{|c|}{ COCINA TRADICIONAL } & \multicolumn{6}{|c|}{ COCINA MEJORADA } \\
\hline & $\begin{array}{l}T_{\mathrm{g}_{1}} \\
\left({ }^{\circ} \mathrm{C}\right)\end{array}$ & $\begin{array}{c}E_{1} \\
\text { (Wh) }\end{array}$ & $\left.\begin{array}{c}E_{a} \\
(\text { Whan }\end{array}\right)$ & $\begin{array}{l}\eta \\
(\%)\end{array}$ & $\begin{array}{l}T_{g 1} \\
\left({ }^{\circ} \mathrm{C}\right)\end{array}$ & $\begin{array}{l}T_{g_{22}} \\
\left.{ }^{\circ}{ }^{\circ} \mathrm{C}\right)\end{array}$ & $\underset{(W h)}{E_{1}}$ & $\begin{array}{l}E_{v 1} \\
(W h)\end{array}$ & $\begin{array}{c}E_{e} \\
\text { (wh) }\end{array}$ & (\%) \\
\hline 1 & 58 & 3332 & 540.1 & 16,2 & 30 & 73 & 1481 & 139,7 & 339,9 & 32,4 \\
\hline 2 & 79 & 4628 & 735.6 & 15,9 & 73 & 78 & 2221 & 254,9 & 454,0 & 31,9 \\
\hline 3 & 72.5 & 5553 & 843,9 & 15,2 & 68,5 & 75,5 & 2777 & 318,9 & 527,3 & 30,5 \\
\hline Promedio & 69,8 & 4504 & 706,5 & 15,8 & 57,2 & 75,5 & 2160 & 237,8 & 440,4 & 31,6 \\
\hline
\end{tabular}
térmica de las cocinas tradicional y mejorada.

TABLA N ${ }^{\circ}$ 02: Valores obtenidos para determinar la eficiencia de la cocina tradicional y mejorada.

GRÁFICO No 1: Curva caracteristica del proceso de calentamiento de la cocina mejorada.

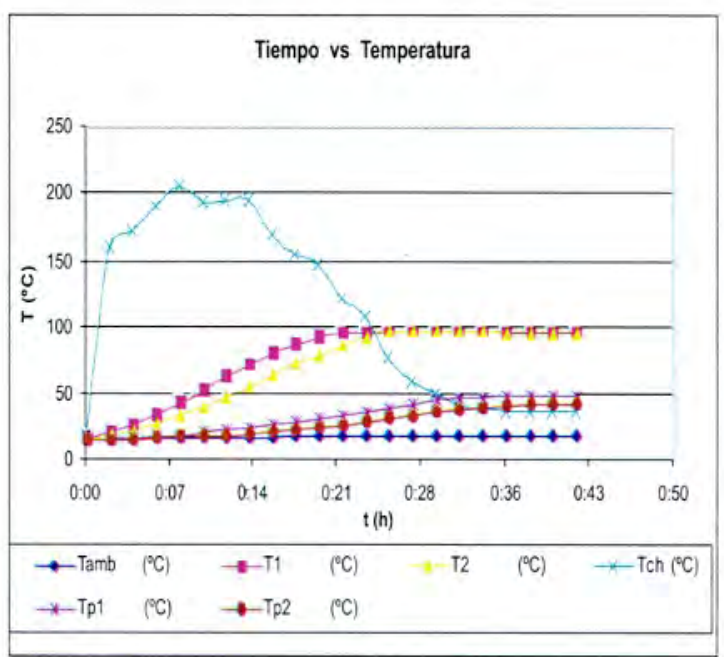


- De acuerdo con las tablas de datos se obtiene que la eficiencia de una cocina tradicional es del $17 \%$ y de una cocina mejorada del $32 \%$, bajo las mismas condiciones de operación.

- De acuerdo con la gráfica mostrada, en la cocina tradicional se alcanzó la máxima temperatura del agua de $98^{\circ} \mathrm{C}$. en 40 minutos mientras que en la cocina mejorada esta temperatura se alcanzó a los 26 minutos aproximadamente.

- De acuerdo con los resultados de la Tabla № 2 , la eficiencia de la cocina mejorada es el doble de la cocina tradicional, por lo que se estima un ahorro del $50 \%$ de la leña que se consume en su utilización.

- En la gráfica queda demostrado que la mayor cantidad de pérdidas de calor se producen por la chimenea, la cual rápidamente llega a altas temperaturas, siendo la mayor de aproximadamente $210^{\circ} \mathrm{C}$, que ocurre en tan sólo siete minutos, pero esto ocurre en forma casi constante durante 21 minutos.

\section{CONCLUSIONES}

1. Las estufas mejoradas de leña son una buena opción de mitigación de la problemática de la contaminación del aire en interiores. Tales estufas utilizan menos leña, emiten menos contaminantes, y no cambian significativamente las costumbres de las comunidades rurales.

2. El uso de cocinas mejoradas puede ser una alternativa muy buena para ahorrar significativamente leña en las zonas rurales, disminuyendo la creciente deforestación y las consecuencias que esta práctica conlleva.

3. El uso de cocinas mejoradas es ya una alternativa para mejorar las condiciones de preparación de desayunos y almuerzos escolares en las zonas rurales, particularmente en la provincia de Tacna.

4. Adicionalmente, seria recomendable evaluar el funcionamiento y las emisiones de las estufas mejoradas después de su utilización en diferentes periodos de tiempo, ya que es necesario darles mantenimiento para mejorar su eficiencia y evitar la fuga de gases durante la combustión.

5. Es urgente, por estas, razones poner en marcha un programa de investigación y desarrollo integral y de largo plazo en el área de la cocción rural, que incluya los siguientes elementos: desarrollo de estufas de leña limpias y eficientes para la cocción rural, determinación de los niveles de exposición de la población a la contaminación de interiores tanto con fogones abiertos como con estufas de leña mejoradas, determinación de los efectos en la salud por la exposición a contaminantes, determinación de los factores de emisión de gases de efecto invernadero asociados a los fogones abiertos y a sus alternativas.

\section{v. BIBLIOGRAFÍA}

ONU - Para la agricultura y la alimentación (1979). Sistemas de almacenamiento de energia para cocinar en las zonas rurales en desarrollo, Lawland.

Gate - GTZ, - Aprovecho Institute (1985), ESTUFAS PARA AHORRAR COMBUSTIBLE. Vieweg \& Sohn, Eschborn, Federal Republic of Germany.

(GIRA), A.C., (2003), El uso de biomasa como fuente de energía en los hogares, efectos en el ambiente y la salud y posibles. soluciones, Informe Final, Michoacan, México. Http:/www.gira.org.mx. Zuñiga, R. (2003), Fogones eficientes. Ministerio de Educación, Departamento Agropecuario, Cuba $\mathrm{Http} /$ /www.cubasolar.cu/biblioteca/energía/Energi a25/HTML/artículo04.htm.

Singer, H.,(2002), Nuevos diseños para cocinas de leña. Asociación Forestal Suiza, Solothurn, Suiza.

Http:/www.fao.org/documents/show_cdr.asp?url_f ile $=/$ docrep $/ \times 5400$ s $/ \times 5400$ s04, htm.

Proyecto Manos Unidas que salva árboles y mejora la calidad de vida (2003), Cocinas mejoradas. Cajama rca, Perú. Http:/www.manosunidas.org.

Nuñez S.,R.(2004), La Turbococina, Antigua Guatemala.http:/www.altavista.com/turbococina.pdf Scout, P. (2004), Una guia Simple para Construir la Estufa Justa, USA. Http:/www.efr.org/apro.

Itacab, (2000), Cocinas Mejoradas de Barro, Lima, Perú.http:/www.itacab.org.

Agencia Cooperante "Carmi Solidarl", (2002), Proyecto Cocinas Mejoradas, Angaraes y Tayacaja, Perú.

Http:/www.ajvilafranca.es/entitats/camisolidari/ Projecuines.html.

Whitfield D./Saavedra R. (2004), Cocinas Ecológicas:Un elemento esencial para una mejor salud en el hogar, Centro de Desarrollo de Energia Solar, Cochabamba, Bolivia. 


\section{ANEXOS:}

Fotografías que muestran el proceso de construcción y evaluación de la cocina mejorada en la Institución Educativa Santa Rita de Calana-Tacna.
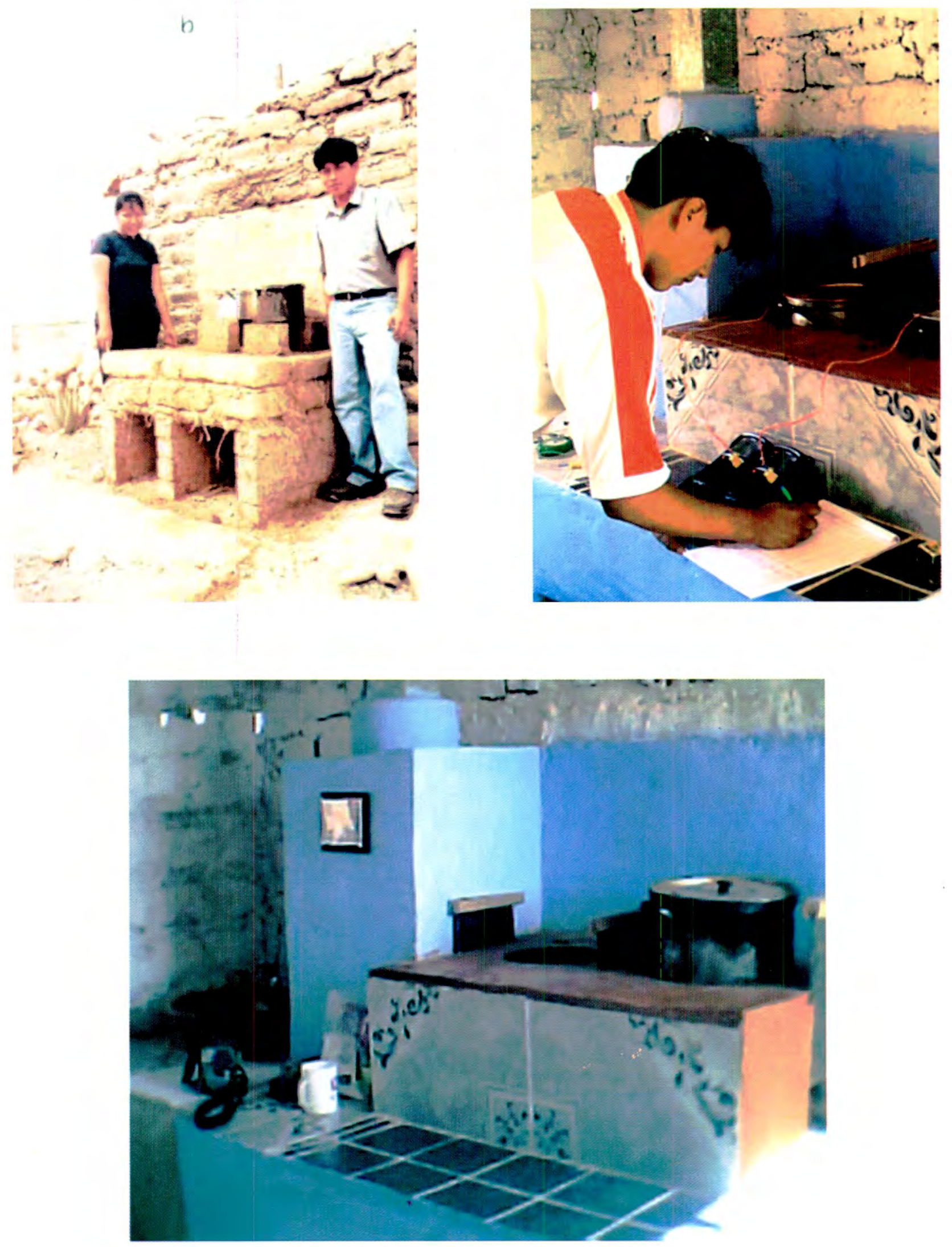